\title{
Evaluation of the COPD Assessment Test and GOLD patient types: a cross-sectional analysis
}

\author{
This article was published in the following Dove Press journal: \\ International Journal of COPD \\ 27 May 2015 \\ Number of times this article has been viewed
}

Jose Luis Lopez-Campos ${ }^{1,2, *}$

Alberto Fernandez-Villar ${ }^{3, *}$

Carmen Calero-Acuña'

Cristina Represas-

Represas ${ }^{3}$

Cecilia Lopez-Ramírez'

Virginia Leiro Fernández ${ }^{3}$

Juan Jose Soler-Cataluña ${ }^{2,4}$

Ricard Casamor ${ }^{5}$

On behalf of the On-Sint

study group

'Unidad Médico-Quirúrgica de Enfermedades Respiratorias, Instituto de Biomedicina de Sevilla (IBiS), Hospital Universitario Virgen del Rocio/Universidad de Sevilla, Sevilla, Spain; ${ }^{2}$ CIBER de Enfermedades Respiratorias (CIBERES), Instituto de Salud Carlos III, Madrid, Spain; ${ }^{3}$ Servicio de Neumología, Instituto de Investigación Biomédica de Vigo (IBIV), Complexo Hospitalario de Vigo, Vigo, Spain; ${ }^{4}$ Servicio de Neumología Hospital Arnau de Vilanova, Valencia, Spain; ${ }^{5}$ Departamento Médico de Novartis Farmacéutica, Barcelona, Spain

*These authors contributed equally to this work

Correspondence: Jose Luis LopezCampos

Hospital Universitario Virgen del Rocio, Avda Manuel Siurot, s/n, 4I0I3 Sevilla, Spain

Email Icampos@separ.es
Background: The COPD Assessment Test (CAT) has been recently developed to quantify COPD impact in routine practice. However, no relationship with other measures in the Global Initiative for Obstructive Lung Disease (GOLD) strategy has been evaluated. The present study aimed to evaluate the relationship of the CAT with other GOLD multidimensional axes, patient types, and the number of comorbidities.

Methods: This was a cross-sectional analysis of the Clinical presentation, diagnosis, and course of chronic obstructive pulmonary disease (On-Sint) study. The CAT score was administered to all participants at the inclusion visit. A GOLD 2011 strategy consisting of modified Medical Research Council scale (MRC) scores was devised to study the relationship between the CAT, and GOLD 2011 axes and patient types. The relationship with comorbidities was assessed using the Charlson comorbidity index, grouped as zero, one to two, and three or more.

Results: The CAT questionnaire was completed by 1,212 patients with COPD. The CAT maintained a relationship with all the three axes, with a ceiling effect for dyspnea and no distinction between mild and moderate functional impairment. The CAT score increased across GOLD 2011 patient types A-D, with similar scores for types B and C. Within each GOLD 2011 patient type, there was a considerably wide distribution of CAT values.

Conclusion: Our study indicates a correlation between CAT and the GOLD 2011 classification axes as well as the number of comorbidities. The CAT score can help clinicians, as a complementary tool to evaluate patients with COPD within the different GOLD patient types.

Keywords: chronic obstructive pulmonary diseases, multidimensional evaluation, comorbidities

\section{Introduction}

COPD is a complex heterogeneous disease where several key factors, including systemic effects and comorbidities, interplay to influence the clinical presentation. ${ }^{1}$ Owing to this, old one-dimensional schemes based on single measurements of lung function impairment, ie, forced expiratory volume in 1 second $\left(\mathrm{FEV}_{1}\right)$, have been abandoned. Accordingly, new multidimensional evaluation proposals have been developed in the context of patientcentered medicine. ${ }^{2}$ In this regard, the most widely accepted proposal is the diagnostic approach proposed by the Global Initiative for Obstructive Lung Disease (GOLD) 2011 , which proposes a three axes scheme, including lung function, exacerbations, and chronic symptoms. ${ }^{3}$ As a part of this multidimensional assessment, the estimation of health status has become one of the pillars of the multidimensional evaluation, providing information about other patient aspects not measured by other available and objective tools, which may complement the clinical information from the patient's perspective. ${ }^{4}$

The COPD Assessment Test (CAT) is a recently developed questionnaire to quantify the impact of COPD on a patient's life and how this changes over time. ${ }^{5}$ Despite 
its short life, this instrument has been validated in several different scenarios, including stable disease, ${ }^{6}$ exacerbations, ${ }^{7}$ association with certain comorbidities, ${ }^{8}$ and after interventions, such as pulmonary rehabilitation. ${ }^{9}$ Thus, the CAT has become one of the main instruments in the evaluation of health status in COPD. Consequently, it has been incorporated into the GOLD strategy. ${ }^{3}$

However, despite these insightful developments, several issues still require attention in order to fully understand the applicability of CAT in COPD. The CAT and modified Medical Research Council scale (MRC) ${ }^{10}$ scores have both been described to reflect disease status and are used equivalently in the GOLD strategy. However, there is a lack of agreement between these scores, and the evaluation of a particular patient may vary depending on the score and the threshold values used. ${ }^{11}$ In addition, there is a considerable variability of the CAT scores among the different GOLD patient types, which has not been fully described. However, by showing the distribution of the CAT values in the different GOLD patient types, more comprehensive information about the potential contribution of the CAT score in the multidimensional evaluation of COPD can be analyzed. Finally, although a close relationship has been described for CAT and comorbidities, ${ }^{8}$ the relationship between the CAT score and the number of comorbidities is controversial. ${ }^{12}$

The Clinical presentation, diagnosis, and course of chronic obstructive pulmonary disease (On-Sint) study was an observational, nationwide, real-life, study performed in Spain between 2011 and 2013, which aimed at evaluating clinical conditions upon diagnosis of COPD and their influence on the progression of the disease. ${ }^{13}$ The present study was a crosssectional analysis of the inclusion visit of the On-Sint cohort and aimed to describe the variability of the CAT values within each GOLD patient type, to evaluate the relationship of the CAT with other GOLD multidimensional axes, and to study its relationship with the number of comorbidities.

\section{Methods}

The On-Sint study was an observational, nationwide, real-life cohort study, in which patients diagnosed with COPD were recruited between December 2011 and April 2013 by primary care (PC) and secondary care (SC) physicians. The methodology has been reported previously. ${ }^{13}$ Briefly, the study recruited consecutive patients aged $>40$ years who were smokers or ex-smokers with a history of $>10$ pack-years; diagnosed with COPD as reported in their medical records; with a complete clinical history of respiratory symptoms; able to complete the CAT questionnaire, and who gave their written informed consent were selected to participate in the study. Ethical approval was granted by the Institutional Review Board from Servicio Gallego de Salud (registry number 2011/359). In order to record real-life clinical behavior of the participant doctors, the only exclusion criterion considered in the study protocol was participation in any other clinical trial at the time of inclusion. In addition, in order to make a real-life evaluation, patients were recruited by PC and SC physicians, with no matching for sex, age, lung function, or any clinical features. Sample size was calculated according to the prevalence and the degree of underdiagnosis of COPD in Spain. ${ }^{14}$ A planned total of 1440 patients with COPD were done to constitute a sample of $0.1 \%$ of the study population, assuming that $10 \%$ of patients would have invalid information. Although a uniform distribution across all regions of the country was planned, the selection of participant investigators was voluntary, with no intention of a representative sampling.

During the inclusion visit, patients underwent a prospective clinical evaluation, spirometry, and completed a CAT questionnaire. The clinical information at the time of the inclusion visit was recorded in a standardized questionnaire and included sociodemographic information, risk factors for COPD (tobacco and other toxic inhalants), presence of chronic symptoms, including: dyspnea (measured by the MRC), chronic cough, and chronic sputum production, number of exacerbations and hospital admissions in the previous year, comorbidities measured using the Charlson comorbidity index, and pharmacological and nonpharmacological treatments for COPD. For the present study, the GOLD definition of exacerbation was adopted, ie, an acute event characterized by a worsening of the patient's respiratory symptoms that is beyond normal day-to-day variations and leads to a change in medication. ${ }^{15}$

Spirometry measurements included pre- and postbronchodilator tests, when available, including forced vital capacity (FVC) and $\mathrm{FEV}_{1}$, as both absolute values and as percentage predicted, and the ratio of $\mathrm{FEV}_{1} / \mathrm{FVC}$ as percentage, carried out at each participating center.

The CAT questionnaire was administered to all participants at the inclusion visit. The questionnaire was selfadministered or administered by the investigator in case of any reading, writing, or sight difficulties. If there were more than two questions unanswered, the questionnaire was considered invalid.

\section{Statistical computations}

Although cases were recruited by respiratory and primary care physicians, all patients were also being followed up by 
general practitioners, so the comparison between PC and SC physicians was impractical and would draw confounding results. Consequently, all patients were analyzed together. Absolute and relative frequencies for categorical questions were used to describe variables. Quantitative data were expressed as mean (standard deviation). To study the relationship between the CAT and GOLD 2011 groups, a modified GOLD 2011 was considered only with the MRC values, as in the previous studies. ${ }^{16,17}$ Comparison of the CAT score in terms of spirometric functional impairment, exacerbation rate, MRC scores, and the number of comorbidities was performed with the analysis of variance (ANOVA) test, using the Welch test in case of nonequivalency of variances, and further analyzed with the Bonferroni correction to detect the differences between categories. The Bonferroni correction is an adjustment made to $P$-values when several dependent or independent statistical tests are being performed simultaneously on a single data set. The results were plotted as bar graphs indicating the mean value, with whiskers indicating the $95 \%$ confidence intervals. Finally, we performed a multivariate linear regression analysis to evaluate which GOLD axis or comorbidities corrected by age and sex were closely associated with CAT. A $P$-value $<0.05$ was considered significant.

\section{Results}

During the study period, 1,264 patients were included in the study. Of these, $50(4.0 \%)$ patients were excluded for not fulfilling the inclusion criteria. Thus, the sample of the On-Sint cohort comprised 1,214 patients with COPD, of which 857 (70.6\%) were recruited by $\mathrm{PC}$ and 357 patients (29.4\%) by SC physicians. The number of cases per region in the country is summarized in Table S1. For the present analysis, two additional patients were excluded for not having completed the CAT questionnaire. Thus, the final sample size for the present analysis was 1,212 patients (Figure 1). None of the CAT questionnaires had missing answers. The characteristics of the study patients are summarized in Table 1: they were predominantly males, with mean age 66 years, $26.2 \%$ were current smokers, with a high number of comorbidities and a mean Charlson comorbidity index of 1.6, and with 2.3 exacerbations in the previous year. The mean CAT value in whole cohort was 18.3 (7.6) points.

\section{CAT and GOLD 20I I}

The CAT score correlated with the degree of lung function impairment, dyspnea, and the exacerbation rate. The CAT scores significantly changed with the spirometric severity. The differences among the spirometric impairment groups are depicted in Figure 2. The CAT score increased from 14.6 (5.9) in those with $\mathrm{FEV}_{1}>80 \%$ to 25.8 (6.2) for those with $\mathrm{FEV}_{1}<30 \%$ (Figure 2). All changes in $\mathrm{FEV}_{1}$ impairment classification were associated with a significant change in the CAT score except for the change between the categories 1 and 2 .

The relationship between the CAT score and the MRC score is depicted in Figure 3. A progressive increase in the

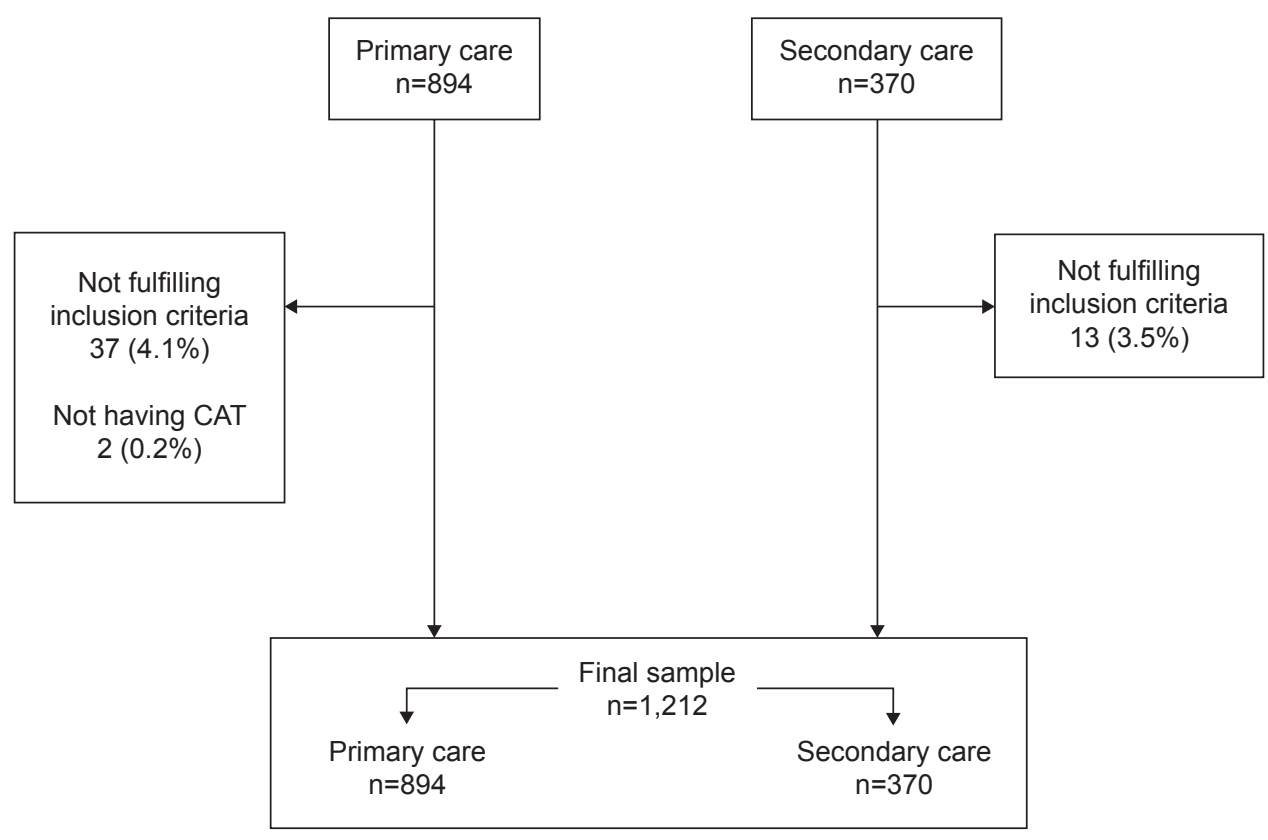

Figure I Flow chart of patients included in the study. Abbreviation: CAT, COPD Assessment Test. 
Table I Baseline characteristics of the patients at the inclusion visit

\begin{tabular}{ll}
\hline & Average (n=I,2 I 2) \\
\hline Age (years) & $66.4(9.7)$ \\
Male sex (n) & $955(78.8)$ \\
Current smokers (n) & $318(26.2)$ \\
Tobacco history (pack-years) & $36.3(20.8)$ \\
Body mass index (kg/m $\left.{ }^{2}\right)$ & $27.7(4.1)$ \\
Comorbidities (Charlson comorbidity index) & $1.6(1.5)$ \\
Dyspnea (MRC) & $1.5(0.8)$ \\
Chronic sputum production (n) & $876(72.3)$ \\
Exacerbations in the previous year (n) & $2.3(1.9)$ \\
Spirometry: FVC (\%) & $74.5(19.5)$ \\
Spirometry: FEV $(\%)$ & $61.6(20.2)$ \\
\hline
\end{tabular}

Note: Data expressed as mean (standard deviation) or absolute (relative) frequencies depending on the nature of the variable.

Abbreviations: FEV , forced expiratory volume in I second; FVC, forced vital capacity; MRC, modified Medical Research Council scale.

CAT score was also shown between the different MRC scores, except for the change between 3 and 4, suggesting a ceiling effect. The CAT score changed from 13.1 (7.8) to 26.1 (7.6), for patients with MRC score 0 and 4, respectively (Figure 3).

The relationship between the CAT score and the number of exacerbations is depicted in Figure 4. For this GOLD axis, the CAT score increased as the number of exacerbations increased. The CAT score changed from 13.5 (7.2) for those with no exacerbations in the previous year to 22.5 (7.5) for those with four or more exacerbations (Figure 4).

The relationship between the CAT score and the GOLD 2011 staging system is depicted in Figure 5. Interestingly, in terms of CAT scores, all stages were significantly different

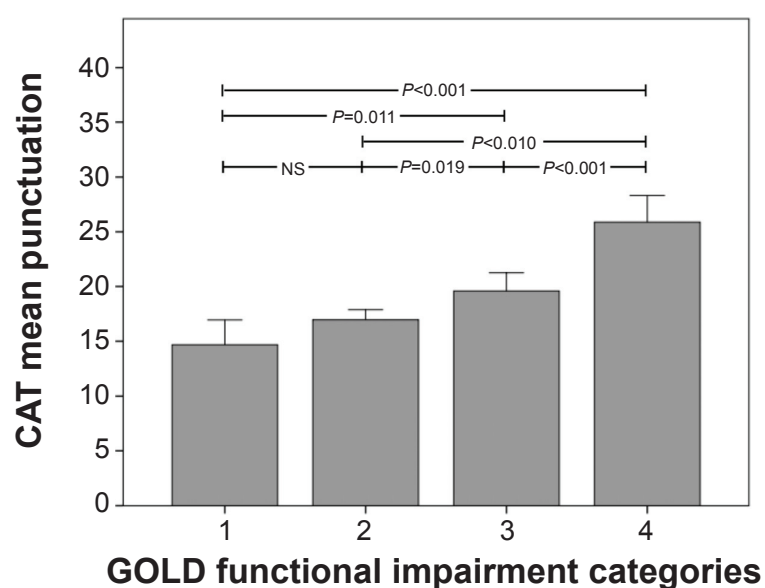

Figure 2 Relationship between spirometric impairment and the CAT score in the On-Sint cohort.

Note: Whiskers represent $95 \%$ confidence intervals.

Abbreviations: CAT, COPD Assessment Test; GOLD, Global Initiative for Obstructive Lung Disease; NS, not significant; On-Sint, Clinical presentation, diagnosis, and course of chronic obstructive pulmonary disease study.

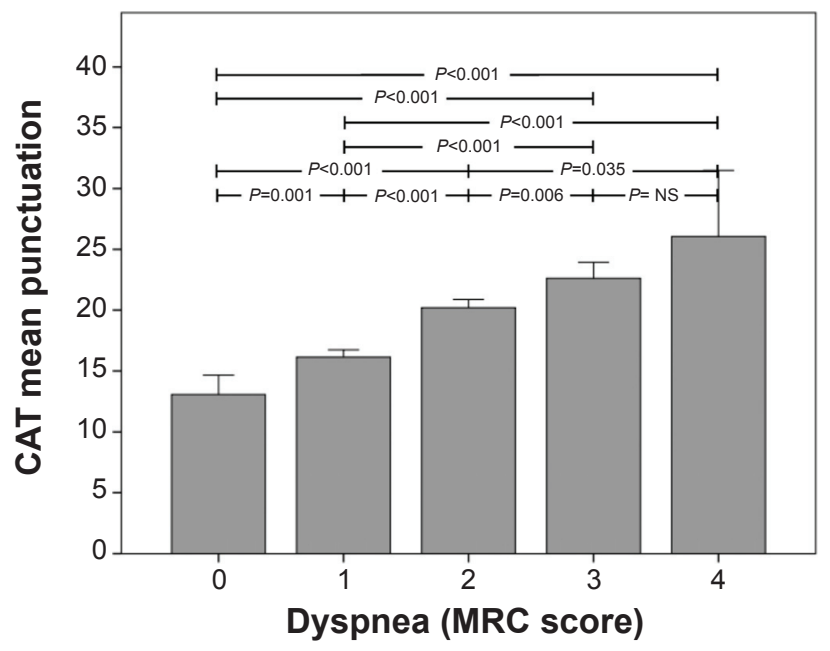

Figure 3 Relationship between the MRC dyspnea score and the CAT score, in the On-Sint cohort.

Note: Whiskers represent $95 \%$ confidence intervals.

Abbreviations: CAT, COPD Assessment Test; MRC, modified Medical Research Council scale; NS, not significant; On-Sint, Clinical presentation, diagnosis, and course of chronic obstructive pulmonary disease study.

from each other, except types B and C. When the CAT scores were represented within each GOLD 2011 type, we observed a considerably wide distribution width, with values ranging from 0 to 40 (Figure 6). Mean values were 12.7 (6.2) for type A, 18.2 (6.8) for type B, 17.6 (7.0) for type C, and 21.8 (6.9) for type $\mathrm{D}$, respectively.

\section{Comorbidities}

The relationship between the CAT score and the comorbidities is depicted in Figure S1. The increase in CAT score was positively correlated to the number of comorbidities and

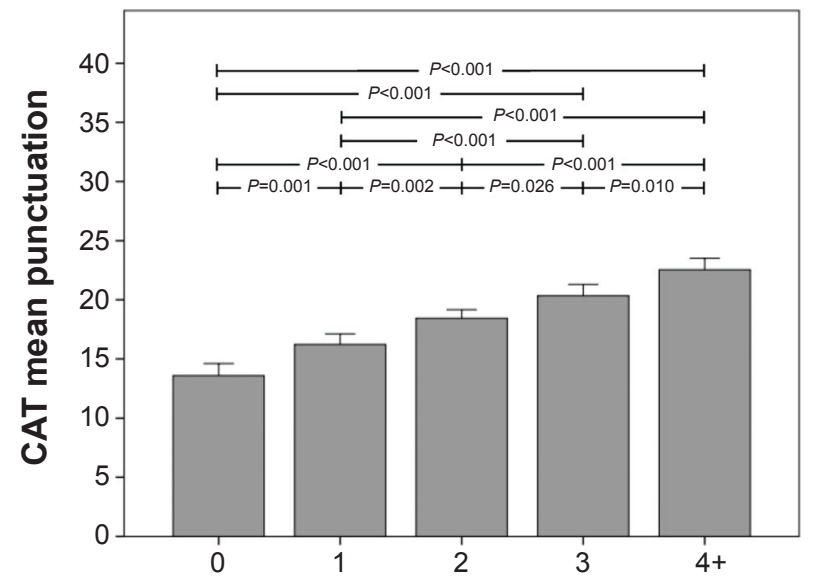

Number of exacerbations in the previous year

Figure 4 Relationship between the number of exacerbations and the CAT score, in the On-Sint cohort.

Note: Whiskers represent $95 \%$ confidence intervals.

Abbreviations: CAT, COPD Assessment Test; On-Sint, Clinical presentation, diagnosis, and course of chronic obstructive pulmonary disease study. 


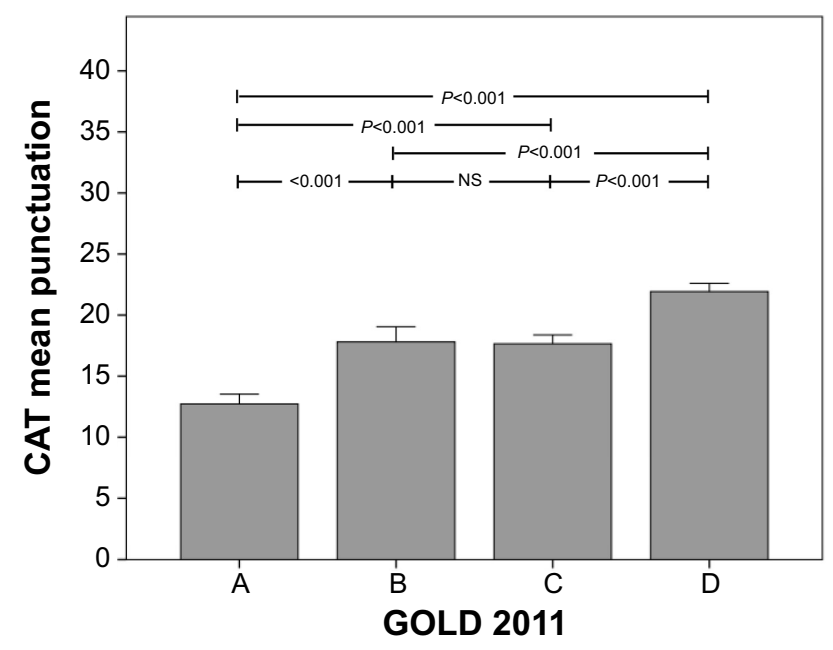

Figure 5 Relationship between the GOLD 20I I staging system and the CAT score, in the On-Sint cohort.

Note: Whiskers represent $95 \%$ confidence intervals. $A=$ low risk, less symptoms; $B=$ low risk, more symptoms; $C=$ high risk, less symptoms; $D=$ high risk, more symptoms. Abbreviations: CAT, COPD Assessment Test; GOLD, Global Initiative for Obstructive Lung Disease; NS, not significant; On-Sint, Clinical presentation, diagnosis, and course of chronic obstructive pulmonary disease study. could be grouped into $0,1-2,3-4$, and $>4$ according to the significance between groups. The CAT score changed from 15.7 (6.9) for those with no comorbidities to 23.1 (8.0) for those with four or more comorbidities. The distribution of the CAT score among patients with different comorbidities is summarized in Table S2.

\section{Multivariate analysis}

The results of the multivariate analysis are presented in Table 2. All the three GOLD axes and the number of comorbidities had correlation with the CAT score. Among them, dyspnea and $\mathrm{FEV}_{1}$ were more importantly associated. Comorbidities seem to play a minor role.

\section{Discussion}

The present study describes the CAT score in a real-life sample of patients with COPD, and analyses the distribution of these values against the GOLD 2011 axes and the
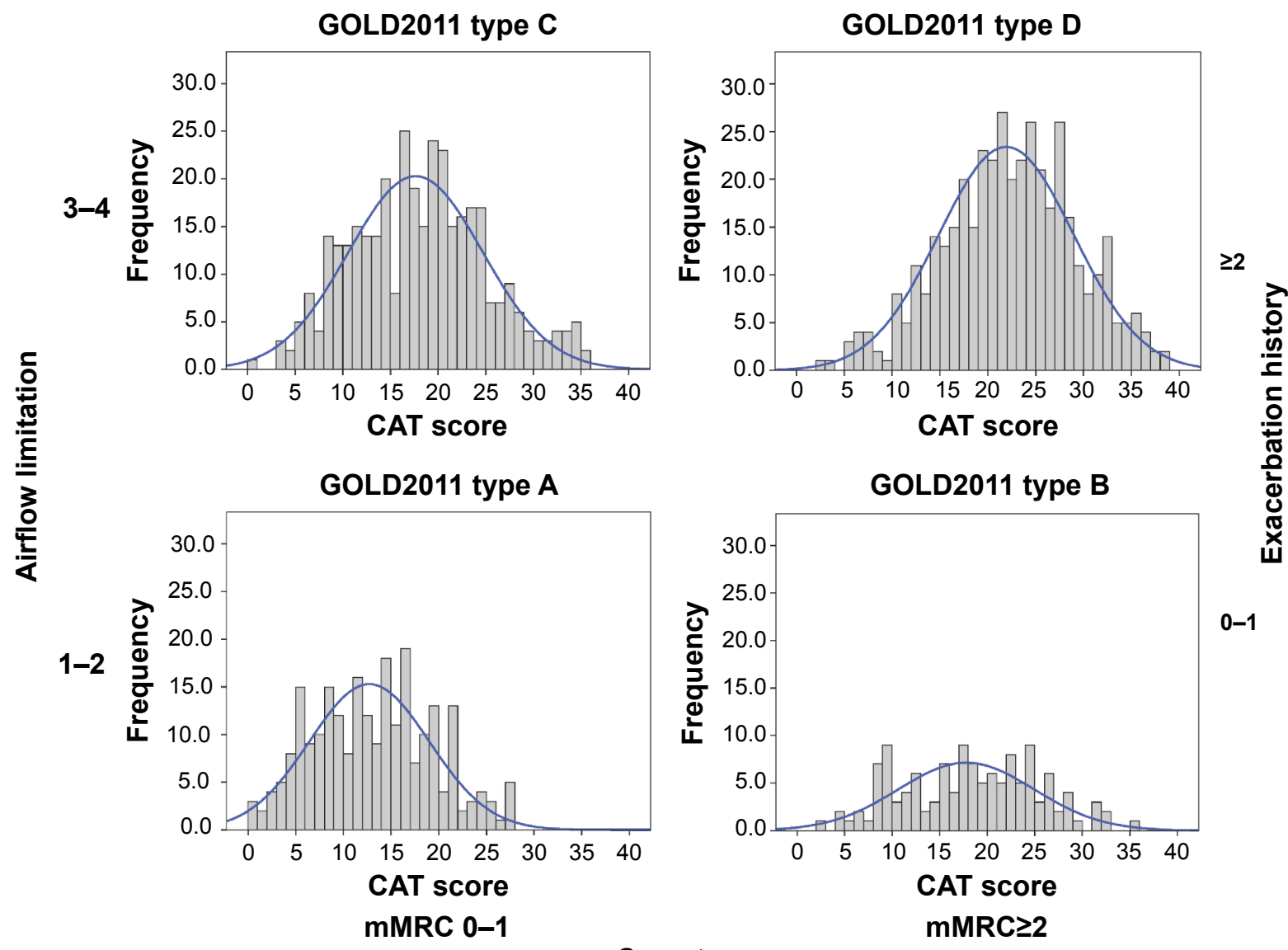

\section{Symptoms}

Figure 6 Distribution of the CAT score among the different modified GOLD 20I I classification.

Abbreviations: CAT, COPD Assessment Test; GOLD, Global Initiative for Obstructive Lung Disease; mMRC, modified Medical Research Council scale. 
Table 2 Multivariate linear regression analysis to describe associations of GOLD axes and comorbidities with CAT

\begin{tabular}{|c|c|c|c|c|}
\hline & \multicolumn{2}{|c|}{ Crude } & \multicolumn{2}{|c|}{ Adjusted* } \\
\hline & $\beta$ & $95 \% \mathrm{Cl}$ & $\beta$ & $95 \% \mathrm{Cl}$ \\
\hline Dyspnea (MRC) & 3.380 & $2.896-3.865$ & 2.500 & $1.688-3.312$ \\
\hline Exacerbations (n) & 2.086 & $1.784-2.389$ & 1.555 & $1.016-2.095$ \\
\hline Spirometric impairment $\left(\mathrm{FEV}_{1}\right)$ & 3.401 & $2.369-4.433$ & 2.194 & $1.235-2.153$ \\
\hline Comorbidities (Charlson comorbidity index) & 1.899 & $1.568-2.229$ & 0.654 & $0.072-1.237$ \\
\hline
\end{tabular}

Note: *Corrected also by age and sex.

Abbreviations: CAT, COPD Assessment Test; Cl, confidence interval; FEV ${ }_{1}$, forced expiratory volume in I second; GOLD, Global Initiative for Obstructive Lung Disease; MRC, modified Medical Research Council scale.

number of comorbidities. Collectively, our data reflects a good association within these dimensions of the disease in spite of a considerable variability within each GOLD type. This suggests that the CAT score could be a good complementary marker for patient evaluation along with MRC, exacerbations, and lung function, as suggested in some clinical guidelines, ${ }^{1}$ helping to refine patient evaluation beyond GOLD types.

\section{Strengths and limitations of this study}

The main strength of this trial is that it was a real-life study, with a large sample size at the national level, where new information about the role of CAT in categorizing of COPD patients was provided. However, several limitations should be taken into consideration to adequately interpret the results of our study. First, this was a cross-sectional analysis, and no prospective follow up of CAT was available. Other studies have evaluated the longitudinal changes in CAT values, indicating a variability in the CAT scores among patients with stable COPD similar to that for the MRC scale score. ${ }^{18}$ Second, the standardized questionnaire used was limited in the number of variables, to make the project affordable. Consequently, several variables, including disease phenotypes or exercise capacity, were not evaluated. Future trials will have to consider other potential variables associated with CAT.

\section{Interpretation of findings in relation to previously published work}

The findings of the present study are in line with previous publications. The Predictive Ability of CAT in Acute Exacerbations of COPD (PACE) study evaluated 495 patients from outpatient clinics in 19 hospitals in Australia, People's Republic of China, Korea, and Taiwan, and found that the baseline CAT score, categorized into four 10-point groups, showed a strong prediction for time to first exacerbation, and modest prediction for any exacerbation or moderate-to-severe exacerbations in the next 6 months. ${ }^{19}$ In Japan, 85 patients with COPD from a hospital outpatient clinic showed strong correlations of CAT with airflow obstruction, dyspnea, exercise tolerance, prognostic index, and oxygenation. ${ }^{20} \mathrm{~A}$ recent systematic review evaluated the psychometric properties of the CAT questionnaire and observed a relationship between the CAT and several clinical outcomes, but not for parameters such as sex, age, smoking status, and comorbidities. ${ }^{21}$ The most relevant study evaluated 882 consultations from 165 PC physicians in the United Kingdom but was carried out with standardized patients with COPD, played by trained actors. The authors concluded that although CAT aids physician assessment of COPD, it does not appear to improve detection of non-COPD symptoms and comorbidities. ${ }^{22}$ Consequently, one strength of our study was that it provided an updated evaluation of the CAT score according to the GOLD 2011 strategy and comorbidities, in a large sample of patients with COPD.

One interesting finding is that despite the reasonably good correlation between CAT and the different GOLD axes, the CAT values between the GOLD 2011 patient types B and C were not different. This is an interesting finding, in line with previous publications on the prognostic capacity of GOLD $2011 .{ }^{16,23}$ In the Evaluation of COPD Longitudinally to Identify Predictive Surrogate End-points (ECLIPSE) study, the authors found that after 3 years of follow up, GOLD 2011 had an impact on all-cause mortality but with no differences between types B and C. ${ }^{23}$ However, when this follow up was done for a longer period of time, all four patient types had a different prognostic impact, as reported by the Collaborative Cohorts to Assess Multicomponent Indices of COPD in Spain (COCOMICS) study. ${ }^{16}$ Interestingly, the COCOMICS and the ECLIPSE studies did not include CAT results; therefore, their GOLD 2011 strategy is also based only on the MRC scores, as in the present study. In addition, the publication of the GOLD 2011 strategy for the management of COPD has shown the discrepancies between the three axes to categorize patients with COPD. The initial studies indicated that there was a mismatch between the MRC and CAT scores for the 
evaluation of individual patients. ${ }^{24}$ Interestingly, different cutoff values were proposed to unify the evaluation of both the scales. ${ }^{11}$ However, although this is valid for a cohort of patients, this discrepancy may still persist in individual patients. ${ }^{25}$ Altogether, as a consequence and according to our results, one potential application of the GOLD 2011 strategy could be to primarily use only the MRC scale and then use the CAT score to further refine the patient type categorization within one GOLD patient type, as suggested by other national guidelines. ${ }^{1}$

The relationship between the CAT score and the number of comorbidities has yielded contradictory results in recent studies. Although previous study has found that a high CAT score may alert the clinician to a higher likelihood of certain comorbidities, such as gastroesophageal reflux and depression, ${ }^{8}$ a recent systematic review including 36 studies and up to 6,469 patients concluded that the CAT score was not statistically different for different comorbidities. ${ }^{21}$ Further, a recent study in Italy found that the relationship between the CAT and comorbidities was not as relevant and that only comorbidities of metabolic and neurological nature showed some correlation with the CAT score, though with very low correlation indices. ${ }^{12}$ The association is relevant because these diseases may coexist unrecognized and also may remain underdiagnosed, yet they are known to have a profound prognostic impact. ${ }^{26}$ In the present study, we have highlighted an increasing CAT score according to comorbidities grouped as zero, one to two, and three or more comorbidities in the Charlson comorbidity index. However, the Charlson comorbidity index does not record all the relevant comorbidities with an impact on health status. Other comorbidities indexes may yield different results. In this regard, the COTE index ${ }^{26}$ and the Functional Comorbidity Index ${ }^{27}$ have not been explored.

\section{Implications for future research, policy, and practice}

The present study provides new information on the use of the CAT in a large sample of patients. According to our results, clinicians can rely on the CAT as a complementary tool to evaluate COPD patients beyond that proposed by the GOLD guidelines. However, there are still key issues to be defined regarding the use of the CAT, such as the minimum clinically relevant difference, the cutoff points for different types of patients, and, more importantly, how to modify treatment according to the score in individual cases. Future research should address these questions, to ensure that the CAT is a necessary tool in daily clinical practice.

\section{Conclusion}

In summary, the results of our study indicate the relationship between the CAT and the GOLD 2011 classification as well as the number of comorbidities. Accordingly, the CAT score seems to be a valid tool for a complementary evaluation of patients with COPD, rather than having a central role to classify different patient types, since it has a wide variability and is influenced by several confounding factors. Consequently, the CAT score can help clinicians to refine the evaluation of patients with COPD beyond the different GOLD patient types.

\section{Acknowledgments}

This project was funded by an unrestricted grant from Novartis Farmacéutica, SA, Spain.

The authors thank all investigators of the On-Sint cohort for their invaluable assistance in collecting the data analyzed here, and Novartis Farmacéutica, SA, Spain, for funding the project.

\section{Author contributions}

JLLC, AFV, and RC conceived the study, analyzed the data, and drafted the manuscript. Additionally, JLLC, AFV, and $\mathrm{RC}$ are the guarantors of the paper, taking responsibility for the integrity of the work as a whole. CCA, CRR, CLR, VLF, and JJSC participated in the coordination of the project, and collection and analysis of the data, discussed the results, and contributed to writing of the manuscript.

\section{Disclosure}

The authors report no conflicts of interest in this work.

\section{References}

1. Miravitlles M, Soler-Cataluña JJ, Calle M, et al. Spanish guideline for COPD (GesEPOC). Update 2014. Arch Bronconeumol. 2014;50 Suppl 1: $\mathrm{S} 1-\mathrm{S} 16$.

2. Lopez-Campos JL, Bustamante V, Muñoz X, Barreiro E. Moving towards patient-centered medicine for COPD management: multidimensional approaches versus phenotype-based medicine - a critical view. COPD 2014;11(5):591-602.

3. Vestbo J, Hurd SS, Rodriguez-Roisin R. The 2011 revision of the global strategy for the diagnosis, management and prevention of COPD (GOLD) - why and what? Clin Respir J. 2012;6(4):208-214.

4. Chrystyn H, Small M, Milligan G, Higgins V, Gil EG, Estruch J. Impact of patients' satisfaction with their inhalers on treatment compliance and health status in COPD. Respir Med. 2014;108(2):358-365.

5. Jones PW, Harding G, Berry P, Wiklund I, Chen WH, Kline Leidy N. Development and first validation of the COPD Assessment Test. Eur Respir J. 2009;34(3):648-654.

6. Jones PW, Tabberer M, Chen WH. Creating scenarios of the impact of COPD and their relationship to COPD Assessment Test (CAT ${ }^{\mathrm{TM}}$ ) scores. BMC Pulm Med. 2011;11:42.

7. Feliz-Rodriguez D, Zudaire S, Carpio C, et al. Evolution of the COPD Assessment Test score during chronic obstructive pulmonary disease exacerbations: determinants and prognostic value. Can Respir J. 2013; 20(5):e92-e97. 
8. Miyazaki M, Nakamura H, Chubachi S, et al; Keio COPD Comorbidity Research (K-CCR) Group. Analysis of comorbid factors that increase the COPD assessment test scores. Respir Res. 2014;15:13.

9. Dodd JW, Marns PL, Clark AL, et al. The COPD Assessment Test (CAT): short- and medium-term response to pulmonary rehabilitation. COPD. 2012;9(4):390-394.

10. Fletcher CM, Elmes PC, Fairbairn AS, Wood CH. Significance of respiratory symptoms and the diagnosis of chronic bronchitis in a working population. Br Med J. 1959;2(5147):257-266.

11. Jones PW, Adamek L, Nadeau G, Banik N. Comparisons of health status scores with MRC grades in COPD: implications for the GOLD 2011 classification. Eur Respir J. 2013;42(3):647-654.

12. Dal Negro RW, Bonadiman L, Turco P. Sensitivity of the COPD assessment test (CAT questionnaire) investigated in a population of 681 consecutive patients referring to a lung clinic: the first Italian specific study. Multidiscip Respir Med. 2014;9(1):15.

13. Fernández-Villar A, López-Campos JL, Represas Represas C, et al. Factors associated with inadequate diagnosis of COPD. On-sint cohort analysis. Int $J$ COPD. In press 2015.

14. Miravitlles M, Soriano JB, García-Río F, et al. Prevalence of COPD in Spain: impact of undiagnosed COPD on quality of life and daily life activities. Thorax. 2009;64(10):863-868.

15. Vestbo J, Hurd SS, Agustí AG, et al. Global strategy for the diagnosis, management, and prevention of chronic obstructive pulmonary disease: GOLD executive summary. Am J Respir Crit Care Med. 2013;187(4): 347-365.

16. Soriano JB, Alfageme I, Almagro P, et al. Distribution and prognostic validity of the new Global Initiative for Chronic Obstructive Lung Disease grading classification. Chest. 2013;143(3):694-702.

17. Vestbo J, Anderson W, Coxson HO, et al; ECLIPSE Investigators. Evaluation of COPD Longitudinally to Identify Predictive Surrogate End-points (ECLIPSE). Eur Respir J. 2008;31(4):869-873.
18. de Torres JP, Marin JM, Martinez-Gonzalez C, et al; COPD History Assessment in Spain (CHAIN) Cohort*. Clinical application of the COPD assessment test: longitudinal data from the COPD History Assessment in Spain (CHAIN) cohort. Chest. 2014;146(1):111-122.

19. Lee SD, Huang MS, Kang J, et al; Investigators of the Predictive Ability of CAT in Acute Exacerbations of COPD (PACE) Study. The COPD assessment test (CAT) assists prediction of COPD exacerbations in high-risk patients. Respir Med. 2014;108(4):600-608.

20. Horita N, Yomota M, Sasaki M, et al. Evaluation of the chronic obstructive pulmonary disease assessment test in Japanese outpatients. Clin Respir J. 2014;8(2):213-219.

21. Gupta N, Pinto LM, Morogan A, Bourbeau J. The COPD assessment test: a systematic review. Eur Respir J. 2014;44(4):873-884.

22. Gruffydd-Jones K, Marsden HC, Holmes S, et al. Utility of COPD Assessment Test (CAT) in primary care consultations: a randomised controlled trial. Prim Care Respir J. 2013;22(1):37-43.

23. Agusti A, Edwards LD, Celli B, et al; ECLIPSE Investigators. Characteristics, stability and outcomes of the 2011 GOLD COPD groups in the ECLIPSE cohort. Eur Respir J. 2013;42(3):636-646.

24. Price DB, Baker CL, Zou KH, Higgins VS, Bailey JT, Pike JS. Realworld characterization and differentiation of the Global Initiative for Chronic Obstructive Lung Disease strategy classification. Int J Chron Obstruct Pulmon Dis. 2014;9:551-561.

25. López Campos JL, Calero C. Questionnaires in multidimensional assessment of chronic obstructive pulmonary disease: two sides of the same coin. Arch Bronconeumol. 2014;50(7):265-266.

26. Divo M, Cote C, de Torres JP, et al; BODE Collaborative Group. Comorbidities and risk of mortality in patients with chronic obstructive pulmonary disease. Am J Respir Crit Care Med. 2012;186(2):155-161.

27. Groll DL, To T, Bombardier C, Wright JG. The development of a comorbidity index with physical function as the outcome. J Clin Epidemiol. 2005;58(6):595-602. 


\section{Supplementary materials}

Table SI Distribution of the included cases among Spanish regions, in the complete On-Sint cohort

\begin{tabular}{|c|c|c|c|}
\hline & $\begin{array}{l}\text { Primary care } \\
(n=857)\end{array}$ & $\begin{array}{l}\text { Secondary care } \\
(n=357)\end{array}$ & $\begin{array}{l}\text { Total } \\
(n=I, 2 \mid 4)\end{array}$ \\
\hline Andalusia & $155(68.6)$ & $71(31.4)$ & 226 \\
\hline Aragon & $23(85.2)$ & $4(14.8)$ & 27 \\
\hline Asturias & $16(100)$ & $0(0)$ & 16 \\
\hline Balearic Islands & $33(100)$ & $0(0)$ & 33 \\
\hline Basque country & $34(73.9)$ & $12(26.1)$ & 46 \\
\hline Canary Islands & $50(65.8)$ & $26(34.2)$ & 76 \\
\hline Cantabria & II (57.9) & $8(42.1)$ & 19 \\
\hline Castilla La Mancha & $31(52.5)$ & $28(47.5)$ & 59 \\
\hline Castilla Leon & 40 (59.7) & $27(40.3)$ & 67 \\
\hline Catalonia & $127(70.2)$ & $54(29.8)$ & 181 \\
\hline Extremadura & $23(74.2)$ & $8(25.8)$ & 31 \\
\hline Galicia & $80(74.1)$ & $28(25.9)$ & 108 \\
\hline La Rioja & $4(100)$ & $0(0)$ & 4 \\
\hline Madrid & $83(65.9)$ & $43(34.1)$ & 126 \\
\hline Murcia & $23(85.2)$ & $4(14.8)$ & 27 \\
\hline Navarre & $15(78.9)$ & $4(2 I . I)$ & 19 \\
\hline Valencia & $109(73.2)$ & $40(26.8)$ & 149 \\
\hline
\end{tabular}

Notes: Data expressed as absolute (relative) frequencies. Percentages refer to the total number of cases in each region.

Abbreviation: On-Sint, Clinical presentation, diagnosis, and course of chronic obstructive pulmonary disease study.

Table S2 Relationship between the different comorbidities in the Charlson comorbidity index and the CAT score

\begin{tabular}{lllc}
\hline & Comorbidity absent & Comorbidity present & P-value* \\
\hline Cardiovascular diseases (any) & $17.1(7.1)$ & $21.4(7.8)$ & $<0.00 I$ \\
Acute myocardial infarction & $18.1(7.4)$ & $20.2(8.6)$ & 0.010 \\
Heart failure & $17.5(7.2)$ & $23.7(7.7)$ & $<0.00 \mathrm{I}$ \\
Peripheral arterial disease & $17.9(7.5)$ & $21.8(7.1)$ & $<0.00 \mathrm{I}$ \\
Cerebrovascular disease & $18.2(7.5)$ & $19.9(8.2)$ & $0.08 \mathrm{I}$ \\
Hemiplegia & $18.3(7.6)$ & $16.7(3.5)$ & $0.68 \mathrm{I}$ \\
Dementia & $18.2(7.5)$ & $20.8(8.3)$ & 0.175 \\
Connective tissue disease & $18.3(7.6)$ & $18.1(6.7)$ & 0.899 \\
Gastroduodenal ulcer & $18.0(7.5)$ & $21.1(7.8)$ & $<0.00 \mathrm{I}$ \\
Hepatic diseases (any) & $18.1(7.5)$ & $22.4(7.4)$ & $<0.00 \mathrm{I}$ \\
Diabetes (any) & $17.3(7.5)$ & $20.2(7.6)$ & $<0.00 \mathrm{I}$ \\
Chronic renal failure & $18.2(7.5)$ & $21.5(8.1)$ & $0.04 \mathrm{I}$ \\
Neoplasms (any) & $18.2(7.5)$ & $19.5(8.9)$ & $0.29 \mathrm{I}$ \\
\hline
\end{tabular}

Notes: Data expressed as mean (standard deviation) of CAT punctuation. *Calculated by unpaired $t$-test.

Abbreviation: CAT, COPD Assessment Test. 


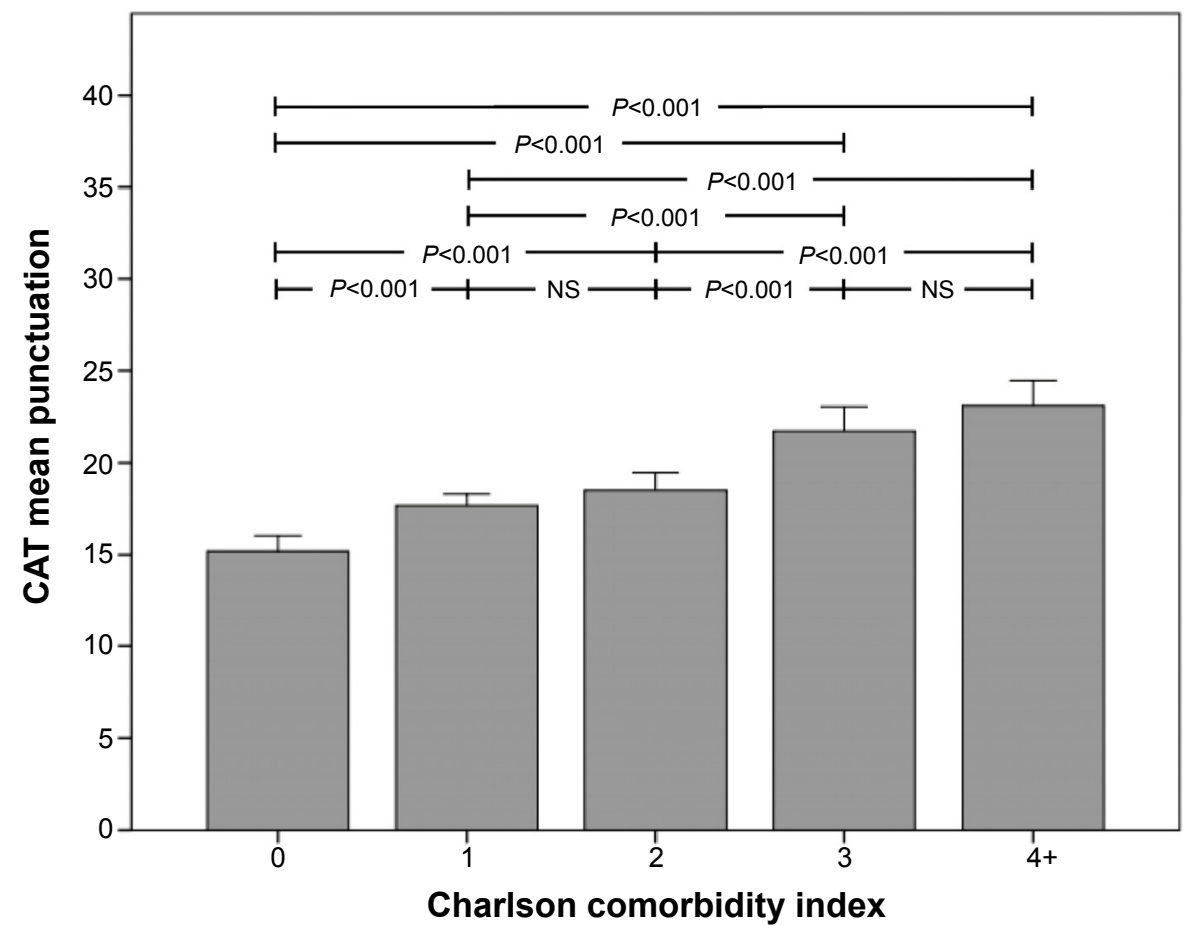

Figure SI Relationship between the Charlson comorbidity index and the CAT score in the On-Sint cohort. Note: Whiskers represent $95 \%$ confidence intervals.

Abbreviations: CAT, COPD assessment test; NS, not significant; On-Sint, Clinical presentation, diagnosis, and course of chronic obstructive pulmonary disease study.

\section{Publish your work in this journal}

The International Journal of COPD is an international, peer-reviewed journal of therapeutics and pharmacology focusing on concise rapid reporting of clinical studies and reviews in COPD. Special focus is given to the pathophysiological processes underlying the disease, intervention programs, patient focused education, and self management protocols.
This journal is indexed on PubMed Central, MedLine and CAS. The manuscript management system is completely online and includes a very quick and fair peer-review system, which is all easy to use. Visit http://www.dovepress.com/testimonials.php to read real quotes from published authors. 